\title{
The Mediating Roles of Disgust Sensitivity and Danger Expectancy in Relation to Hand Washing Behaviour
}

\author{
Susan J. Thorpe \\ University of Surrey, Guildford, UK \\ Julie Barnett \\ Brunel University, Uxbridge, UK \\ Katy Friend and Kate Nottingham \\ University of Surrey, Guildford, UK
}

Background: Recent interest in the role of vulnerability factors in obsessional washing has suggested that disgust sensitivity, danger expectancy and health anxiety may be of interest. Aims: This study explores the differential impact of these factors on both behavioural and cognitive measures of washing behaviour and is based on a replication of the Jones and Menzies (1997) experiment, during which participants immersed their hands in a noxious compound while rating themselves on a range of measures: the time they subsequently took to wash their hands was measured and danger expectancies were found to be the best predictor of this. Method: The present study added measures of disgust sensitivity and health anxiety to this experimental methodology while removing factors they found to be of little import to compulsive washing. Thirty non-clinical participants took part. Results: Results confirmed that disgust sensitivity was related to the behavioural measure of washing time, but that this relationship was almost entirely mediated by the danger expectancy concerning judgements of severity of consequent disease. However, a different pattern emerged when the outcome measure was questionnaire based: danger expectancy was not at all related to this. Disgust sensitivity mediated the relationship between health anxiety and scores on a questionnaire measure of washing compulsions. Interestingly, these scores were not related to the behavioural measure of washing time. Conclusions: The implications of these relationships to the further development of subtypes of Obsessive Compulsive Disorder (OCD) are discussed.

Keywords: Disgust, danger expectancy, contamination fear, compulsive washing, obsessive compulsive disorder.

Reprint requests to S. J. Thorpe, Department of Psychology, Faculty of Arts and Human Sciences, University of Surrey, Guildford GU2 7XH, UK. E-mail: s.thorpe@surrey.ac.uk 


\section{Introduction}

Several psychological factors have been identified as contributing to the triggers and maintaining factors of obsessional behaviour: these include responsibility (Rachman, 1993; Rhéaume, Ladouceur, Freeston and Letarte, 1995; Salkovskis and Forrester, 2002; Salkovskis, Shafran, Rachman and Freeston, 1999; Salkovskis et al., 2000); self-efficacy (Williams and Watson, 1985) control (Moulding, Kyrios and Doron, 2007); perfectionism (Coles, Frost, Heimberg and Rhéaume, 2003; Frost and Steketee, 1997; Reiss, 1991; Reiss and McNally, 1985; Tallis, 1996); anticipatory anxiety and avoidance (Reiss, 1991; Reiss and McNally, 1985) and of particular relevance to this study; inflated expectations of danger (Jones and Menzies, 1997).

Although previously conceptualized as a singular disorder, evidence is growing in support of the notion that OCD is a heterogeneous group of disorders which, as well as commonalities, may have different aetiologies, maintaining factors and responses to treatments, (Cougle, Wolitzky-Taylor, Lee and Telch, 2007; Sookman, Abramowitz, Calamari, Wilhelm and McKay, 2005; Thorpe, Patel and Simonds, 2003). This process of separation began as early as 1974 when Rachman (1974) singled out obsessional slowness for attention, and was further refined by Rachman and Hodgson (1980) when they distinguished between washers and checkers and more recently by Mataix-Cols (Mataix-Cols, 2006; Mataix-Cols, RosarioCampos and Leckman, 2005). However, the differential importance of the factors, noted above, to the posited subtypes of OCD has begun to be explored only relatively recently, and is still being debated (Clark, 2005; Radomsky and Taylor, 2005). This paper sets out to examine factors that may be of particular relevance to washing compulsions.

In an interesting study involving a behavioural outcome measure of washing behaviour, Jones and Menzies (1997) highlighted the influence of danger expectancies concerning illness beliefs (judgements concerning both the likelihood of contracting a disease and the severity of disease once caught) on the formation of washing compulsion. They used a range of selfreport measures and a behavioural avoidance test (BAT) during which participants with OCD immersed their hands in disgusting material: time spent washing their hands was measured immediately afterwards. They found no evidence that factors previously accepted as important in the aetiology of OCD such as responsibility beliefs, perfectionist ideas or perceptions of self-efficacy were related to time spent washing, and further argued that inflated responsibility appraisals are rarely found in obsessional washers and may in fact only be a secondary problem in people with OCD in general. They suggested that both types of danger expectancy were equally important in relation to urge to wash and time spent washing. In a followup study, Menzies, Harris, Cumming and Einstein (2000) examined these again in tandem with a responsibility manipulation (personally responsible or other person responsible) and concluded that those in the personally responsible group differed from the other group in their ratings of the severity-related (but not likelihood-related) danger expectancy, which they suggest may go on to influence washing and checking behaviour. However, neither of these studies included a measure of disgust sensitivity in their models, which subsequent studies have found to be of interest in relation to washing compulsions. Charash and McKay (2002) and Muris et al. (2000), for instance, found disgust sensitivity to be strongly associated with OCD, particularly to obsessions related to contamination fear. Mancini, Gragnani and D’Olimpio (2001), in a questionnaire study, found that washing and checking behaviours (but not impulses and rumination) were best predicted by a measure of disgust sensitivity, while 
Thorpe et al. (2003) also found some support for the view that disgust sensitivity, along with health anxiety, may play a particular role in the washing subtype of OCD. More specifically, both health anxiety and disgust sensitivity were related to the amount of distress associated with washing behaviour and with the judgements of frequency of washing behaviour, although health anxiety was the most powerful predictor of the former (associated distress) and disgust sensitivity of the latter (judgement of frequency). This however was a questionnaire study and did not include a consideration of the role that danger expectancies might play. Tolin, Woods and Abramowitz (2006) suggested that checking, ordering, and washing were also all associated with disgust sensitivity, even when controlling for anxiety and depression. Disgust sensitivity also appears to be one of the factors able to enhance the relationship between the propensity to feel disgust and fear of contamination (Cisler, Olatunji, Sawchuk and Lohr, 2008), while fear of contamination has also been related to mental pollution (Fairbrother, Newth and Rachman, 2005; Rachman, 2007). Olatunji (2009) has also suggested that disgust propensity and sensitivity are vulnerability factors for the aspect of excessive health anxiety concerned with sensitivity to changes in the body, (body vigilance) which may impact upon beliefs about illness and contamination. Finally, Cougle et al. (2007) note that in their treatment group for sub-clinical washers, urge to wash after behavioural avoidance tasks was related to levels of anxiety in those with illness related threat beliefs. However, for those with non-illness threat beliefs, levels of disgust were related to urge to wash, not anxiety. They did not however use standardized measures of disgust sensitivity or health anxiety.

Taken together, these findings tentatively suggest that exploring specific elements of the OCD experience may be useful The preliminary evidence cited above indicates that, with regard to compulsive washing, there is a case to be made for further exploration of the role of danger expectancies, individual sensitivity to disgust and to health anxiety, thus drawing together variables that previous research suggests may be aetiologically interesting but which have not been tested all together in relation to both behavioural and self-report measures of washing behaviour/contamination fear.

This paper therefore sets out to explore the posited relationship between disgust sensitivity, health anxiety, danger expectancies and cognitive and behavioural measures of hand washing, in vivo, within a non-clinical group. It builds specifically on the work by Jones and Menzies (1997) and Thorpe et al. (2003) and will do this by replicating the experimental methodology described by Jones and Menzies (1997), by removing the elements that they found to be redundant (responsibility and perfectionism), retaining those factors related to danger expectancies concerning illness beliefs, and adding factors previously identified as being implicated in self-reported levels of obsessional hand washing: namely, measures of selfreported disgust sensitivity and health anxiety (Thorpe et al., 2003). Anxiety itself has not been found to be of particular interest in this model but will be measured in the event of it being a confounding variable in this study. This methodology was chosen because it has an observable behavioural outcome measure as well as a valid and reliable questionnaire measure, and thus possibly greater ecological validity. The use of a non-clinical sample can be justified because previous research has shown the utility of recruiting non-clinical participants to study obsessions (Cougle et al., 2007; Olatunji, Abramowitz, Williams, Connolly and Lohr, 2007; Olatunji, Cisler, Deacon, Connolly and Lohr, 2007; Olatunji, Forsyth and Cherian, 2007; Olatunji, Lohr, Sawchuk and Tolin, 2007)

This paper addresses the following questions: Are disgust sensitivity, danger expectancies and health anxiety related to measures of washing behaviour? Are they related to a 
standardized self-report measure of OCD washing concerns? If so, which, if any, of these factors best predicts washing behaviour and scores on the self report measure?

\section{Method}

\section{Participants}

Thirty-five undergraduate students volunteered to take part in the study in return for course credits, 14 of whom were male and 21 female. Their ages ranged from 21 years to 25 years, with a mean of 21.7 ( $S D$ 0.88). All but one of the participants defined themselves as White British, with the remaining person identifying as Indian. All participants took part in the BAT (see below).

Thirty-two out of 35 participants were able to tolerate the BAT for the maximum time. The three remaining kept their hands immersed for 2, 5 and 10 seconds respectively. As there were so few who kept their hands in the dirt for such a short time and everyone else completed 5 minutes, their data were removed from the analyses and this variable was not examined further. Two further participants were removed from the analysis as their scores were above two standard deviations of the mean on the main outcome measure (time taken to wash). This left us with 13 males and 17 females, all of whom defined themselves as White British.

\section{Materials/apparatus}

A bin $36 \mathrm{~cm}$ high and $32 \mathrm{~cm}$ in diameter was used for the BAT. The recipe for its contents was that used by Jones and Menzies (1997): a mixture of potting soil, animal hair, raw meat, and household food scraps.

\section{Measures}

The Beck Anxiety Inventory (BAI; Beck and Steer, 1990). This scale has 21 items relating to common physical symptoms of anxiety. The respondent is asked to rate how much they have been bothered by each item in the past week, on a scale of 0 to 3 , and to indicate how much they have been bothered by that symptom in the last month. The items are then summed to obtain their score, ranging from 0-63. This scale was included as general anxiety was a possible confound. This scale has a Cronbach's $\alpha$ of .87

Obsessive Compulsive Inventory - Revised (OCI-R; Foa et al., 2002). The OCI-R is an 18item self-report measure of obsessive compulsive symptoms designed for use with clinical and non-clinical samples. Each item is rated on a 5-point Likert scale (ranging from "not at all" to "extremely") according to how much the respondent is distressed or bothered (during the past month) by various obsessions and compulsions. It provides an overall score as well as scores on six subscales (checking, hoarding, neutralizing, obsessing, ordering, and washing). The OCI-R has excellent psychometric properties (Foa et al., 2002; Thorpe et al., 2003) Cronbach's $\alpha$ in this sample was .92. Alphas for the subscales were as follows: washing $\alpha=.65$, obsessing $\alpha=.78$, hoarding $\alpha=.77$, ordering $\alpha=.92$, checking $\alpha=.68$, neutralizing $\alpha=.71$. The washing scale has a relatively low reliability score that, according to Nunnally (Nunnally, 1978), is usually acceptable above $\alpha=.7$. However, the Cronbach's alpha is dependent on the number of items in the scale, such that the fewer the items the lower 
the Cronbach's tends to be. Therefore, with only three items in these subscales, these alphas are acceptable.

The Disgust Sensitivity scale (DS; Haidt, McCauley and Rozin, 1994). This is a 32-item scale measuring disgust sensitivity across seven domains: animals, body products, death, envelope violations (injuries, wounds), food, hygiene and sex. An eighth subscale (sympathetic magic) is included in the overall score. The questionnaire is divided into two parts with the first half requiring an answer as to whether the respondent agrees with the statement, ranging from 1- Strongly disagree (very untrue about me) to 4 - Strongly agree (very true about me). The second half of the questionnaire requires a rating of how disgusting they would find each of the experiences listed on a scale of 1 to 4 where 1 is "not disgusting at all" and 4 is "very disgusting". Scores can run from 32 to 128 . Cronbach's $\alpha$ in this sample was .88.

Whitely index/Health Anxiety scale (HA; Pilowsky, 1967). This is a health anxiety questionnaire consisting of 14 statements that are answered by circling a number on a 5point scale indicating how much the statement "is true for you". Anchors are "not at all", "a little bit", "moderately", "quite a bit" and "a great deal". The Whitely index score is found by summing the responses. Cronbach's $\alpha$ in this sample was .90 .

Danger Expectancies (DEL and DES). These were visual analogue scales, with a range from 0 to 100, identical to those used in the Jones and Menzies (1997) experiment. Danger Expectancy Likelihood (DEL) of catching a disease: "How likely is it that you will catch a disease or become ill?" Where possible response range from 0 (not at all possible) to 100 (certain to occur). Danger Expectancy of Severity (DES) of disease or illness: "If you caught a disease or became ill how severe would your illness be?" Ratings were on a scale from 0 (no noticeable symptoms-minor illness) to 100 (terminal illness-death certain).

Anticipatory anxiety; anticipatory disgust; perceived self-efficacy; level of state disgust during BAT; level of state anxiety during BAT; state urge to wash during BAT. These were visual analogue scales with a range from 0 to 100 , identical to those used in the Jones and Menzies (1997) study except where concerned with disgust ratings, which were specific to this study. Anticipatory anxiety: "How anxious would you feel if asked to place your hands in the container up to wrist level?" Ratings were on a scale from 0 (no anxiety) to 100 (worst anxiety you can imagine). Anticipatory disgust: "How disgusted would you feel if asked to place your hands in the container up to wrist level?" Ratings were on a scale from 0 (no disgust) to 100 (most disgust they could imagine). Perceived self-efficacy: "How long do you think you could comfortably keep your hands in the container?" Response was in estimated number of seconds. State disgust: "How disgusted do you feel?" Ratings were on a scale from 0 (not at all disgusted) to 100 (extremely disgusted). State anxiety: "How anxious do you feel?" Ratings were on a scale from 0 (not at all anxious) to 100 (worst anxiety you can imagine). State urge to wash: "How strongly do you feel like washing your hands?" Ratings were on a scale from 0 (no urge) to 100 (extreme urge).

\section{Procedure}

All participants were individually put through the study by the third and fourth authors. There were three distinct parts to the experiment: before, during and after the BAT. 
Pre-BAT. Participants filled in the BAI, OCI-R, DS and HA. Procedure and instructions then followed the Jones and Menzies (1997) procedure and instructions exactly. First they were told "the bin in front of you contains potting soil, animal hair, food scraps and raw meat. In a moment I am going to ask you some questions about this container. There are no right or wrong answers. Each question is designed to be answered quickly and is not meant to be thought about too much". Participants were not at this stage told that they would shortly be immersing their hands in the mixture. They were then asked to estimate the level of anxiety they would feel if they had to put their hands in the mixture before them, using the visual analogue scales related to anticipatory anxiety, disgust, and perceived self-efficacy.

Peri-BAT. Following this, the participants were given the following instructions: "I would like you to place your hands in the container and keep them there for 5 minutes. During this time I am going to ask you some questions about this experience. Again, there are no right or wrong answers. Each question is designed to be answered quickly and is not meant to be thought about too much. If, at some point during the task, you feel unable to keep your hands in the bin any longer you may remove them". Once the participants' hands were immersed in the bin, they were shown the Likert scales and, once a minute, were asked to move their hands around a little to maximize contact with unpleasant items and were asked the two questions related to danger expectancies and three questions related to their levels of disgust, anxiety and urge to wash. These were asked every minute, up to the maximum of 5 minutes, when the BAT ended.

Post-BAT. Participants were told that they could now go and wash their hands. This was timed without their being aware of it.

\section{Results}

\section{Overview}

Data exploration began with examination of the data for assumptions of normality. All scores were normally distributed apart from the scores on the washing subset of the OCI-R, which were somewhat positively skewed. This was subsequently transformed using $\log 10$. Relationships between disgust sensitivity, danger expectancies (likelihood and severity), health anxiety and washing behaviour were explored. As all participants (apart from those removed from analysis) had kept their hands in the soil for the whole 300 seconds, this factor was not further examined in relation to any other variable.

\section{Pre-BAT and peri-BAT scores}

Table 1 shows the group mean scores. With a high average urge to wash hands of 69.6, and medium scores for disgust, participants clearly felt uncomfortable during this task. Unexpectedly, they also spent significantly longer washing their hands after the BAT than did the OCD washers in the Jones and Menzies (1997) study. This comparison may not be valid given the possible differences in situational factors in which this study was undertaken, but is retained for interest. Participants spent an average of 2 minutes 12 seconds washing compared to 1 minute 31 seconds in the OCD study. A one sample $t$-test was carried out to compare these scores: and the student group spent significantly longer washing their hands 
Table 1. Summary of data

\begin{tabular}{llrrrr}
\hline & & \multicolumn{4}{c}{ Range } \\
\cline { 5 - 6 } & & Mean $n=30$ & $\min$ & $\max$ & \multicolumn{1}{c}{$S D$} \\
\hline Pre-BAT & Anxiety inventory & 14.73 & 0 & 25 & 6.36 \\
& Health anxiety & 22.97 & 16 & 36 & 5.55 \\
& Disgust sensitivity & 80.37 & 56 & 104 & 11.62 \\
& OCI-R total & 13.70 & 3 & 55 & 10.85 \\
& OCI-washing subscale & 1.73 & 0 & 7 & 1.81 \\
& Anticipatory disgust & 47.17 & 10 & 80 & 19.64 \\
& Anticipatory anxiety & 36.73 & 0 & 90 & 24.72 \\
& Perceived self-efficacy (seconds) & 194.67 & 5 & 300 & 120.33 \\
Peri- BAT & & & & \\
& Danger likelihood of disease/illness & 7.24 & 0 & 38 & 9.15 \\
& Danger severity of disease/illness & 8.93 & 0 & 30 & 8.03 \\
& State disgust & 48.09 & 4 & 94 & 23.80 \\
& State anxiety & 30.89 & 0 & 82 & 23.47 \\
& State urge to wash hands & 69.18 & 2 & 100 & 24.97 \\
& Time in dirt (0-300 seconds) & 300 & 300 & 300 & 0.00 \\
Post- BAT & Washing time (seconds) & 131.90 & 30 & 236 & 56.07 \\
\cline { 5 - 6 } & & & &
\end{tabular}

than did the OCD (Jones and Menzies 1997) group $(t=4.304, p<.005)$. This is unexpected, especially in the light of the Jones and Menzies OCD washers group being significantly more anxious (an average of 52.1 in the OCD group compared to 30.89 for the students: $t=-4.949$, $p<.0001$ ) as rated on the state anxiety visual analogue scales during the task. They were very similar in their scores on the urge to wash scale (OCD group washers 68.3).

Relationships between disgust sensitivity (DS), danger expectancies relating to likelihood and severity, (DEL and DES), health anxiety (HA) and washing time

A correlation analysis was conducted with DS, DEL, DES, HA and washing time. General anxiety was also included in case it was a confounding variable. The Danger Expectancy Likelihood (DEL) of catching a disease was not related to the behavioural outcome measure of washing time. In contrast, Danger Expectancy Severity (DES) of disease was related to washing time.

Disgust sensitivity was related to both DES and to washing time, but not to DEL. Table 2 shows these results in detail. State anxiety, state disgust and state urge to wash (measures taken while participants had their hands in the soil) were also correlated with washing time.

It is clear from the correlation matrix that the two danger expectancies are working in different ways, with DEL being unrelated to any of the other variables, and DES related to all variables except scores on the OCI-R washing subscale. It is also noteworthy that the scores on the Disgust Sensitivity Scale are related to all variables (with the one exception noted above) while health anxiety, though related to several variables, is not related to washing time or to either of the danger expectancy variables. Anxiety scores are only related to health anxiety, disgust sensitivity scores and the state anxiety score visual analogue scale. As they are not related to any of the outcome variables, anxiety scores will not be discussed further. 
Table 2. Correlation coefficients

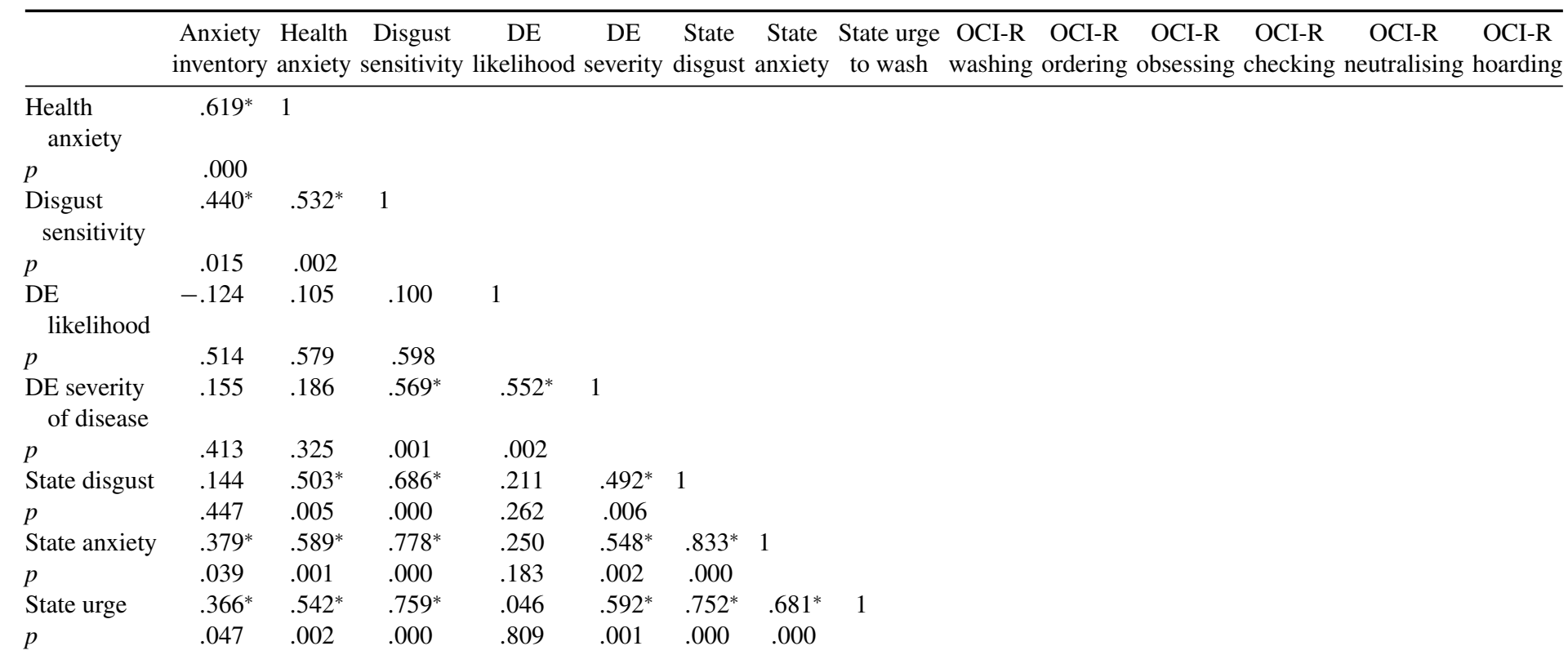




\begin{tabular}{|c|c|c|c|c|c|c|c|c|c|c|c|c|c|c|}
\hline OCI-R washing & .229 & $.546^{*}$ & $.627^{*}$ & .065 & .227 & $.506^{*}$ & $.453^{*}$ & $.544^{*}$ & 1 & & & & & \\
\hline$p$ & .223 & .002 & .000 & .733 & .227 & .004 & .012 & .002 & & & & & & \\
\hline OCI-R ordering & .236 & $.496^{*}$ & $.597^{*}$ & .133 & .237 & .295 & .312 & $.438^{*}$ & $.730^{*}$ & 1 & & & & \\
\hline$p$ & .208 & .005 & .000 & .483 & .207 & .114 & .094 & .015 & .000 & & & & & \\
\hline $\begin{array}{l}\text { OCI-R } \\
\text { obsessing }\end{array}$ & $.465^{*}$ & $.693^{*}$ & .328 & .168 & .163 & $.404^{*}$ & $.524^{*}$ & $.370^{*}$ & $.504^{*}$ & $.370^{*}$ & 1 & & & \\
\hline$p$ & .010 & .000 & .076 & .376 & .389 & .027 & .003 & .044 & .005 & .044 & & & & \\
\hline OCI-R checking & $.575^{*}$ & $.694^{*}$ & .334 & .221 & .256 & .272 & $.440^{*}$ & $.389^{*}$ & $.455^{*}$ & $.420^{*}$ & $.824^{*}$ & 1 & & \\
\hline$p$ & .001 & .000 & .071 & .241 & .172 & .146 & .015 & .034 & .011 & .021 & .000 & & & \\
\hline $\begin{array}{l}\text { OCI-R } \\
\text { neutralizing }\end{array}$ & .277 & $.614^{*}$ & .182 & .196 & .132 & .310 & .208 & $.407^{*}$ & $.630^{*}$ & $.558^{*}$ & $.586^{*}$ & $.669^{*}$ & 1 & \\
\hline$p$ & .139 & .000 & .336 & .299 & .488 & .096 & .270 & .026 & .000 & .001 & .001 & .000 & & \\
\hline OCI-Rhoarding & $.402^{*}$ & $.715^{*}$ & $.363^{*}$ & .216 & .179 & .157 & .325 & .306 & $.411^{*}$ & $.536^{*}$ & $.641^{*}$ & $.740^{*}$ & $.469^{*}$ & 1 \\
\hline$p$ & .028 & .000 & .049 & .251 & .343 & .409 & .079 & .100 & .024 & .002 & .000 & .000 & .009 & \\
\hline Washing time & .255 & .247 & $.508^{*}$ & .291 & $.593^{*}$ & $.632^{*}$ & $.631^{*}$ & $.584^{*}$ & .249 & .146 & .347 & .336 & .283 & -.002 \\
\hline$p$ & .174 & .187 & .004 & .118 & .001 & .000 & .000 & .001 & .184 & .440 & .061 & .070 & .130 & .992 \\
\hline
\end{tabular}

*Correlation is significant. Actual $p$ values are shown. DE Likelihood = Danger Expectancy Likelihood; DE severity $=$ Danger Expectancy; OCI-R washing $=$ Obsessive Compulsive Inventory-Revised washing subscale. 
Of particular interest is the finding that the OCI-R washing subscale scores are not related to the actual washing time behavioural measure.

The hypothesized link between health anxiety and washing time is not supported. Neither is the link between DEL and washing time.

Relationship between perceived self-efficacy, urge to wash, washing time and danger expectancy likelihood and danger expectancy severity

Self-efficacy was measured in terms of the number of seconds participants felt they would be able to keep their hands in the dirt. This was significantly negatively correlated with disgust sensitivity $(r=-.449 . p=.013)$ and average urge to wash $(r=-.624, p<.0001)$ but not with DES $(r=-.316, p=.089)$. The relationship between this measure and the OCI-R washing subscale was significantly negatively correlated $(r=-0.413, p=.023)$ but there was no relationship between self-efficacy and DEL or washing time.

\section{Relationships between disgust sensitivity, danger expectancy severity and washing time}

In line with the other part of the research question, there appears to be strong links between these three variables: disgust sensitivity, DE severity and washing time. In order to explore these relationships in more detail a mediation analysis was carried out (Baron and Kenny, 1986). This method was chosen for two main reasons: the data set was not large enough to use structural equation modelling, and the method of mediation used here is particularly suitable for single-mediator models (MacKinnon and Fairchild, 2009).

Initially the regression coefficient for the relationship between DS and washing time was $2.45(\mathrm{SE}=.786)$ and was significantly different from zero $(p=.0004)$. After controlling for the mediator (DE severity) the regression coefficient for DS was reduced to 1.22 ( $\mathrm{SE}=.879$ ) and was no longer significantly different from zero $(p=.178)$. This suggests that level of DE severity mediated the relationship between the independent variable (DS) and dependent variable (washing time). In order to test this, a Sobel test was carried out (Sobel, 1982). This indicated that there was significant mediation $(z=2.04746, p=.0406)$.

\section{Contribution of disgust sensitivity and health anxiety to OCI washing subtype scores}

As an additional exploration, examining a different washing related outcome measure, and in line with Thorpe et al. (2003), the specific relationship between health anxiety, disgust sensitivity and OCI-R washing scores was explored. (None of the danger expectancies were related to this variable so it was not possible to carry out an identical analysis to that above). Previous research had found differences in the amount of variance explained by DS and HA when distress or frequency scales were used, with disgust implicated as more important in frequency associated with washing scores, and HA in distress related to washing scores. As the OCI-R focuses on the amount of distress experienced, it was expected that Health Anxiety might be mediating the relationship between disgust sensitivity and OCI-R washing subscale scores. A meditational analysis was therefore carried out to check whether HA mediated the effect of DS on OCI-R washing scores.

So, initially the regression coefficient for the relationship between disgust sensitivity and the OCI-R washing score was $.015(\mathrm{SE}=.015)$ and was significantly different from zero 
( $p=<.001)$. After controlling for the mediator (HA) the regression coefficient for DS reduced to $.011(\mathrm{SE}=.004)$ but was still significantly different from zero $(p=0.007)$. HA does not appear to be mediating the relationship.

In order to test whether in fact it was DS that was mediating the relationship between HA and the OCI-R washing score, another mediation analysis was carried out. The regression coefficient for the relationship between HA and OCI-R washing was .026 (SE $=.008$ ) and was significantly different from zero $(p=.002)$. After controlling for the mediator (DS) the regression coefficient reduced to $.013(\mathrm{SE}=.008)$ and was no longer significantly different to zero $(p=.106)$. This suggests that DS mediated the relationship between the independent variable (HA) and dependent variable (OCI-R washing score). In order to confirm this, a Sobel test was carried out (Sobel, 1982). This indicated that there was significant mediation $(z=2.11999, p=.0340)$.

Relationship between danger expectancies, disgust sensitivity, health anxiety, anxiety and measures of subtypes of $O C D$

In order to explore the differential importance of variables, as outlined in the introduction, to subtypes of OCD, the relationship between DEL and DES and subscales of the OCI-R, and to the OCI-R total score were examined. There were no significant relationships found.

The relationship between DS and OCI-R subscale scores was examined. Thorpe et al. (2003) found that DS was correlated with all OCI distress subscales, though most powerfully with the washing distress subscale. In the present study, DS was significantly related to scores on the washing, ordering and hoarding subscales but not to the three other subscale scores of neutralizing, checking and obsessing, although the latter two subtype scores were close to a significant relationship to DS.

In further exploration of differential influences on different obsessional subscales, the general anxiety scores, and health anxiety scores were examined in relation to the OCI-R subscale scores. General anxiety was only significantly related to the checking, obsessing and hoarding subscales, while health anxiety was related to all the subscales. Interestingly, only health anxiety is related to the neutralizing subscale. These figures can be seen in detail in Table 2. It is of interest to note that even with a limited number of participants and a non-clinical participant group, different clusters of relationships between variables begin to emerge.

\section{Discussion}

Research engaging with the emotion of disgust has become exponentially apparent in psychological research over the last 10 years, particularly in relation to anxiety. Some authors have approached this with scepticism and have previously reported evidence both for and against the importance of disgust: for, in obsessions (Thorpe et al., 2003) and against in spider phobia (Thorpe and Salkovskis, 1998) for example. The present study provides more evidence to add to the growing body of literature suggesting that the emotion of disgust may be important when examining correlates of contamination fears and obsessional washing concerns. This study found that DS mediates the relationship between HA and scores on the OCI-R washing subscale but that, when actual behavioural measures of washing are scrutinized, the relationship with HA disappears, the role of disgust sensitivity is demoted 
and danger expectancy severity appears to be more interesting, mediating as it does between DS and washing time.

The initial analysis focused on the relationship between the factors of particular interest to the study: disgust sensitivity, the danger expectancies of likelihood of disease and severity of disease, health anxiety and washing time. In line with the Jones and Menzies (1997) finding, the danger expectancy of severity of disease was related to disgust sensitivity, the visual analogue scales of disgust, anxiety, urge to wash and to washing time. However, the other danger expectancy (likelihood of catching a disease) was not related to the behavioural outcome measure of washing time, nor to any other variable apart from its companion danger expectancy (estimate of severity of disease). This disparity is an echo of the results of the Menzies et al. (2000) study, which found that the severity but not likelihood danger expectancy was related to judgements (in a group with experimentally inflated personal responsibility) for negative outcomes related to washing and checking.

Here, disgust sensitivity, health anxiety and general anxiety were all related to each other. Apart from this, general anxiety was only related to the state anxiety peri-BAT scale while health anxiety was related to all three - anxiety, disgust, and urge to wash - as well as to scores on the OCI-R washing subscale. Washing time was also related to these three variables as well as to the severity danger expectancy and disgust sensitivity, but not to the OCI-R washing subscale. Self-efficacy also showed a mixed pattern of connection, but was not related to washing time: it was negatively related to disgust sensitivity, severity danger expectancy, and average urge to wash and the OCI-R washing subscale. There is therefore an interesting pattern of initial results with different variables related to OCI-R washing scores and to actual washing time, with only disgust sensitivity and three of the measures taken while hands were in the soil related to both the cognitive (OCI-R washing) and behavioural (washing time) washing related measures.

The main focus of the analysis was on the relationship between disgust sensitivity, severity danger expectancy, and washing time. The results in part support the findings of Jones and Menzies (1997) who suggested that danger expectancies were important in relation to washing behaviour in OCD, although in the present study only the danger expectancy concerning the perception of severity of disease was related to washing behaviour. The mediation analysis showed that the relationship between disgust sensitivity and washing time was mediated by the danger expectancy concerning severity measure (what Jones and Menzies call the "verbal, cognitive" element). So someone high in disgust sensitivity might spend longer washing if they believed in the likely severity of any disease caught as a consequence of their hand being immersed in a noxious substance, irrespective of their probability judgement of actually contracting such a disease: the perceived cost associated with an aversive outcome appeared to be related to the amount of time people spent washing (i.e. neutralizing the threat) where the related probability estimate was not. In the formulating treatments for washing subtype of OCD it may therefore be useful to pay particular attention to levels of disgust sensitivity and to this particular cognition.

However, of particular interest here was the finding that the pattern of results was very different when the outcome was measured not by behaviour but by the OCI-R washing subscale. Health anxiety, for example, was not related to washing time but was related to the OCI-R washing subscale. However, our analysis showed that disgust sensitivity mediated the relationship between health anxiety and OCI-R washing. This is different to previous findings by Thorpe et al. (2003) and may be due to the BAT heightening disgust sensitivity or other 
situational factors. (Additionally, the low scale reliability in our sample may also indicate that this finding should be treated with some caution). So someone high in health anxiety may have a high score on the OCI-R subscale if they are also high in disgust sensitivity. Recent research by Olatunji (2009) has found an interesting relationship between disgust propensity and sensitivity and particular aspects of health anxiety (awareness of bodily changes rather than probability of contracting an illness), which may be of relevance here.

The final analyses concerned relationships between the three main measures of disgust sensitivity, health anxiety and general anxiety and the OCD-R subtype scores. This is of interest in the context of a burgeoning general interest in refining understanding of the individual subtypes and identifying differences and commonalities. Again, there was an inconsistent pattern of results, with health anxiety being related to all the subtypes, disgust sensitivity related to washing, ordering and hoarding subtypes, and anxiety related to checking, obsessing and hoarding. All effect sizes were large, which may indicate that, given enough participants, all the relationships between the three emotional states and the six subtypes would have been meaningful. However, there is enough variation to be of interest: hoarding is unusual for example in being the only subtype measure to be related to all the disgust, health anxiety and anxiety measures.

An additional finding of interest was that the participants in the present study spent longer washing their hands than did the participants with a diagnosis of OCD in the previous study. It is unclear why this was the case and any pronouncement as to a likely cause would be purely speculative, for instance, that the present participants were on average 12 years younger than in the previous study and as the majority were female, the length of their nails, and any ornamentation thereon, may need to be taken into account. Additionally, recent public health campaigns (relating to first, bird flu and more recently, swine flu) have focused on the necessity of hygienic behaviours, particularly hand washing. Future research may need to take account of this and attempt an explanation should it occur again. Further clarity should also be sought on the impact of different types of disgust on different types of anxiety. Woody and Tolin (2002), while agreeing that disgust sensitivity could be a predisposing factor in developing an aversion, suggest that it is state rather than trait disgust that impacts on behaviour and that, further, OCD washing is specifically associated with certain domains of disgust - animals and body products. The participants in the present study were informed of the contents of the bin, so this may have had some influence on their responses. Olatunji, Sawchuck, Lohr and de Jong (2004), on the other hand, found contamination fear was related to 7 disgust domains, and concluded that it was predicted by generalized, not domain specific, disgust.

This paper is limited in that it uses non-clinical participants and as such any conclusions drawn are not necessarily generalizable to people with OCD, though Gibbs (1996) has made a case for the use of non-clinical participants in research in OCD and Olatunji has produced numerous papers utilizing non-clinical participants. In addition, these results raise questions about the nature of the relationship between these emotional, behavioural and cognitive factors that cannot be answered by the information available here. Since gathering these data, research into disgust sensitivity has been further refined by consideration of the roles of disgust propensity and disgust sensitivity, with each hypothesized as contributing differentially to measures such as health anxiety (Olatunji, 2009) and other specific fears (Van Overveld, de Jong, Peters, Cavanagh and Davey, 2006). More attention has also begun to be paid to the possible role of anxiety sensitivity (Reiss, Peterson, Gursky and McNally, 1986; Taylor and 
Cox, 1998a, 1998b) while Sookman et al. (2005) have begun the process of trying to match treatment to subtypes. Further research into the processes at work both transdiagnostically and idiodiagnostically in relation to cognitive factors, sensitivity to emotions and washing compulsions would be very useful in the refocusing of effective subtype therapies.

\section{References}

Baron, R. and Kenny, D. (1986). The moderator-mediator variable distinction in social psychological research: conceptual, strategic, and statistical considerations. Journal of Personality and Social Psychology, 51, 1173-1182.

Beck, A. and Steer, R. (1990). Manual for the Beck Anxiety Inventory. San Antonio, TX: Psychological Corporation.

Charash, M. and McKay, D. (2002). Attention bias for disgust. Journal of Anxiety Disorders, 16, 529-541.

Cisler, J. M., Olatunji, B. O., Sawchuk, C. N. and Lohr, J. M. (2008). Specificity of emotional maintenance processes among contamination fears and blood-injection-injury fears. Journal of Anxiety Disorders, 22, 915-923.

Clark, D. (2005). Lumping versus splitting: a commentary on subtyping in OCD. Behavior Therapy, 36, 401-404.

Coles, M., Frost, R., Heimberg, R. and Rhéaume, J. (2003). "Not just right experiences": perfectionism, obsessive-compulsive features and general psychopathology. Behaviour Research and Therapy, 41, 681-700.

Cougle, J., Wolitzky-Taylor, K., Lee, H. and Telch, M. (2007). Mechanisms of change in ERP treatment of compulsive hand washing: does primary threat make a difference? Behaviour Research and Therapy, 45, 1449-1459.

Fairbrother, N., Newth, S. and Rachman, S. (2005). Mental pollution: feelings of dirtiness without physical contact. Behaviour Research and Therapy, 43, 121-130.

Foa, E., Huppert, J., Leiberg, S., Langner, R., Kichic, R., Hajcak, G. and Salkovskis, P. (2002). The Obsessive-Compulsive Inventory: development and validation of a short version. Psychological Assessment, 14, 485-496.

Frost, R. and Steketee, G. (1997). Perfectionism in obsessive-compulsive disorder patients. Behaviour Research and Therapy, 35, 291-296.

Gibbs, N. (1996). Nonclinical populations in research on obsessive-compulsive disorder: a critical review. Clinical Psychology Review, 16, 729-773.

Haidt, J., McCauley, C. and Rozin, P. (1994). Individual differences in sensitivity to disgust: a scale sampling seven domains of disgust elicitors. Personality and Individual Differences, 16, 701-713.

Jones, M. K. and Menzies, R. G. (1997). The cognitive mediation of obsessive-compulsive handwashing. Behaviour Research and Therapy, 35, 843-850.

MacKinnon, D. and Fairchild, A. (2009). Current directions in mediation analysis. Current Directions in Psychological Science, 18, 16.

Mancini, F., Gragnani, A. and D'Olimpio, F. (2001). The connection between disgust and obsessions and compulsions in a non-clinical sample. Personality and Individual Differences, 31, 1173-1180.

Mataix-Cols, D. (2006). Deconstructing obsessive-compulsive disorder: a multidimensional perspective. Current Opinion in Psychiatry, 19, 84.

Mataiz-Cols, D., Rosario-Campos, M. and Leckman, J. (2005). A multidimensional model of obsessive-compulsive disorder. American Journal of Psychiatry, 162, 228.

Menzies, R. G., Harris, L. M., Cumming, S. R. and Einstein, D. A. (2000). The relationship between inflated personal responsibility and exaggerated danger expectancies in obsessive-compulsive concerns. Behaviour Research and Therapy, 38, 1029-1037. 
Moulding, R., Kyrios, M. and Doron, G. (2007). Obsessive-compulsive behaviours in specific situations: the relative influence of appraisals of control, responsibility and threat. Behaviour Research and Therapy, 45, 1693-1702.

Muris, P., Merckelbach, H., Nederkoorn, S., Rassin, E., Candel, I. and Horselenberg, R. (2000). Disgust and psychopathological symptoms in a nonclinical sample. Personality and Individual Differences, 29, 1163-1167.

Nunnally, J. (1978). Psychometric Theory. New York: McGraw-Hill.

Olatunji, B. O. (2009). Incremental specificity of disgust propensity and sensitivity in the prediction of health anxiety dimensions. Journal of Behavior Therapy and Experimental Psychiatry, 40, 230-239.

Olatunji, B. O., Abramowitz, J. S., Williams, N. L., Connolly, K. M. and Lohr, J. M. (2007). Scrupulosity and obsessive-compulsive symptoms: confirmatory factor analysis and validity of the Penn Inventory of Scrupulosity. Journal of Anxiety Disorders, 21, 771-787.

Olatunji, B. O., Cisler, J. M., Deacon, B. J., Connolly, K. and Lohr, J. M. (2007). The Disgust Propensity and Sensitivity Scale-Revised: psychometric properties and specificity in relation to anxiety disorder symptoms. Journal of Anxiety Disorders, 21, 918-930.

Olatunji, B. O., Forsyth, J. P. and Cherian, A. (2007). Evaluative differential conditioning of disgust: a sticky form of relational learning that is resistant to extinction. Journal of Anxiety Disorders, 21, $820-834$.

Olatunji, B. O., Lohr, J. M., Sawchuk, C. N. and Tolin, D. F. (2007). Multimodal assessment of disgust in contamination-related obsessive-compulsive disorder. Behaviour Research and Therapy, 45, 263-276.

Olatunji, B. O., Sawchuk, C. N., Lohr, J. M. and de Jong, P. J. (2004). Disgust domains in the prediction of contamination fear. Behaviour Research and Therapy, 42, 93-104.

Pilowsky, I. (1967). Dimensions of hypochondriasis. The British Journal of Psychiatry, 113, 89.

Rachman, S. (1974). Primary obsessional slowness. Behaviour Research and Therapy, 12, 9-18.

Rachman, S. (1993). Obsessions, responsibility and guilt. Behaviour Research and Therapy, 31, 249278.

Rachman, S. (2007). Unwanted intrusive images in obsessive compulsive disorders. Journal of Behavior Therapy and Experimental Psychiatry, 38, 402-410.

Rachman, S. and Hodgson, R. (1980). Obsessions and Compulsions: New York: Prentice Hall.

Radomsky, A. and Taylor, S. (2005). Subtyping OCD: prospects and problems. Behavior Therapy, 36, 371-379.

Reiss, S. (1991). Expectancy model of fear, anxiety, and panic. Clinical Psychology Review, 11, 141153.

Reiss, S. and McNally, R. J. (1985). Expectancy model of fear. Theoretical Issues in Behavior Therapy, 107-121.

Reiss, S., Peterson, R. A., Gursky, D. M. and McNally, R. J. (1986). Anxiety sensitivity, anxiety frequency and the prediction of fearfulness. Behaviour Research and Therapy, 24, 1-8.

Rhéaume, J., Ladouceur, R., Freeston, M. and Letarte, H. (1995). Inflated responsibility in obsessive compulsive disorder: validation of an operational definition. Behaviour Research and Therapy, 33, 159-169.

Salkovskis, P. and Forrester, E. (2002). Responsibility. In R. O. Frost and G. Steketee (Eds.), Cognitive Approaches to Obsessions and Compulsions: theory, assessment, and treatment (pp. 4561). Amsterdam: Pergamon/Elsevier.

Salkovskis, P., Shafran, R., Rachman, S. and Freeston, M. (1999). Multiple pathways to inflated responsibility beliefs in obsessional problems: possible origins and implications for therapy and research. Behaviour Research and Therapy, 37, 1055-1072.

Salkovskis, P., Wroe, A., Gledhill, A., Morrison, N., Forrester, E., Richards, C., Reynolds, M. and Thorpe, S. (2000). Responsibility attitudes and interpretations are characteristic of obsessive compulsive disorder. Behaviour Research and Therapy, 38, 347-372. 
Sobel, M. (1982). Asymptotic confidence intervals for indirect effects in structural equation models. Sociological Methodology, 13, 290-312.

Sookman, D., Abramowitz, J., Calamari, J., Wilhelm, S. and McKay, D. (2005). Subtypes of obsessive-compulsive disorder: implications for specialized cognitive behavior therapy. Behavior Therapy, 36, 393-400.

Tallis, F. (1996). Compulsive washing in the absence of phobic and illness anxiety. Behaviour Research and Therapy, 34, 361-362.

Taylor, S. and Cox, B. (1998a). Anxiety sensitivity: multiple dimensions and hierarchic structure. Behaviour Research and Therapy, 36, 37-51.

Taylor, S. and Cox, B. (1998b). An expanded anxiety sensitivity index evidence for a hierarchic structure in a clinical sample. Journal of Anxiety Disorders, 12, 463-483.

Thorpe, S. J., Patel, S. P. and Simonds, L. M. (2003). The relationship between disgust sensitivity, anxiety and obsessions. Behaviour Research and Therapy, 41, 1397-1409.

Thorpe, S. J. and Salkovskis, P. M. (1998). Studies on the role of disgust in the acquisition and maintenance of specific phobias. Behaviour Research and Therapy, 36, 877-893.

Tolin, D. F., Woods, C. M. and Abramowitz, J. S. (2006). Disgust sensitivity and obsessive-compulsive symptoms in a non-clinical sample. Journal of Behavior Therapy and Experimental Psychiatry, 37, $30-40$.

Van Overveld, W., de Jong, P., Peters, M., Cavanagh, K. and Davey, G. (2006). Disgust propensity and disgust sensitivity: separate constructs that are differentially related to specific fears. Personality and Individual Differences, 41, 1241-1252.

Williams, S. and Watson, N. (1985). Perceived danger and perceived self-efficacy as cognitive mediators of acrophobic behavior. Behavior Therapy, 16, 136-146.

Woody, S. and Tolin, D. (2002). The relationship between disgust sensitivity and avoidant behavior: studies of clinical and nonclinical samples. Journal of Anxiety Disorders, 16, 543-559. 